\title{
Cyclic compression of the intracranial optic nerve: patterns of visual failure and recovery
}

\author{
Case report \\ L. FRISÉN, ${ }^{1}$ J. SJÖSTRAND, K. NORRSELL, AND S. LINDGREN \\ From the Departments of Ophthalmology and Neurosurgery, University of Göteborg, Sweden
}

SYNOPSIS A patient with a cystic craniopharyngioma below the right optic nerve had several recurrences requiring surgery. Finally the cyst was connected with a subcutaneous reservoir by means of a fine catheter. Symptoms of optic nerve compression recurred more than 50 times during the following year, and were relieved within seconds upon drainage of the reservoir. In each cycle, a drop in visual acuity preceded a measurable change in the visual field. The pattern of field changes was an increasingly severe, uniform depression. Optic nerve ischaemia induced by compression was probably the most important factor causing visual failure in this case.

Compression of an optic nerve by an adjacent mass characteristically causes a slowly progressive loss of vision. Once diagnosed, surgical exploration is usually carried out rather promptly to prevent further visual loss. Thus, there are few opportunities to study systematically the progression of visual failure for any length of time before treatment. Similarly, opportunities to record the early phases of visual recovery after decompression come rarely. The exact moment of surgical relief of compression is easily recorded but because of the circumstances surrounding surgery, formal testing usually has to be postponed for many hours. We know of only one extensively studied case that gives some relevant information. Isayama (1970) has given a description, in Japanese, of a case with a huge craniopharyngioma cyst that pressed on the left intracranial optic nerve. Surgical removal was not possible. Instead, the cyst was connected to a subcutaneous reservoir with the idea of draining the cyst percutaneously if symptoms recurred. Isayama studied two cycles of cyst refilling with respect to optic nerve function, and noted that vision was rapidly restored (within 24 hours) upon drainage. No information was given on the early time-course of visual recovery.

We have had the opportunity to monitor visual

${ }^{1}$ Address for correspondence and reprints: Dr L. Frisén, Ögonkliniken, Sahlgrenska sjukhuset, S-413 45 Göteborg, Sweden.

(Accepted 16 June 1976.) function in a similar case with a small, collapsible craniopharyngioma cyst, where the optic nerve suffered more than 50 cycles of compression and decompression without acquiring signs of progressive damage. Recovery of vision was instantaneous upon drainage of the cyst. Our observations will be described and discussed within the framework of recent advances in axonal physiology.

\section{CASE REPORT}

At the age of 33 years, a mother of three children first noticed visual loss. Examination at her local hospital disclosed an asymmetrical chiasmal syndrome. No changes were seen on plain skull films, but pneumoencephalography demonstrated a suprasellar mass, indenting the third ventricle. Neurosurgical exploration was made at Sahlgrenska sjukhuset in March 1969. A cystic mass was encountered between the optic nerves, dislocating the left nerve laterally, and raising and stretching the right nerve. The mass was removed but for some small capsule fragments below the right optic nerve. Microscopic examination of tumour fragments suggested an epithelial origin of the tumour and with some reservation the pathologist classified it as a craniopharyngioma.

Recovery was uneventful, with complete restoration of central vision in both eyes. There remained a permanent lower nasal cut in the right visual field, and the right optic disc showed slight pallor. The visual 
field of the left eye was considered normal. Colour vision was defective in the right eye (Ishihara test plates).

Symptoms recurred one month after surgery. Further neurosurgical exploration in May 1969 disclosed a cystic tumour below and medial to the right optic nerve. The cyst was removed as completely as possible, and visual function recovered to the preoperative level. Remnants of the cyst capsule caused new recurrences, however. Another attempt to remove the tumour was made in November 1969. At this time capsule remnants were coagulated by local application of Zenker's solution. A fourth operation was performed in January 1970, but on this occasion the cyst was connected to a subcutaneous Ames reservoir behind the right ear by means of silicone tubing (Fig. 1A) with the aim of allowing percutaneous drainage. Postoperative recovery was uneventful, and vision was restored to the same level as after the first procedure.

Symptoms recurred thrice during the following year, each time producing severe visual loss in the right eye. These symptoms were relieved by drainage of up to $3 \mathrm{ml}$ of yellow fluid from the reservoir behind the ear. The rate of cyst filling accelerated, however, and the patient was again admitted for further studies.

Two millilitres of water soluble contrast medium
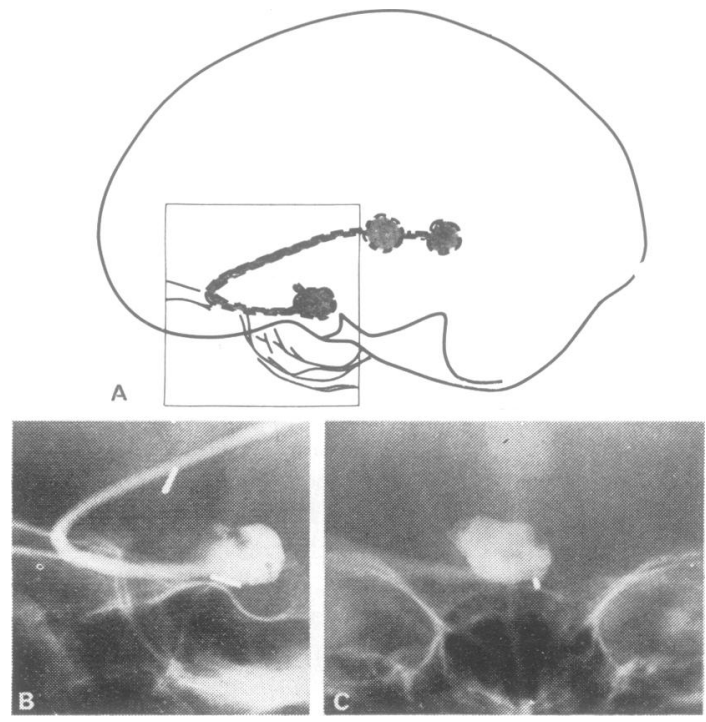

FIG. 1 Appearance of suboptic craniopharyngioma cyst with catheter and reservoir after contrast injection. A: schematic drawing. B: left lateral radiograph. $C:$ frontal radiograph. (meglumine iocarmate, Dimer-X) were injected into the cyst by the reservoir. X-ray studies confirmed the location of the cyst, below and medial to the right optic nerve (Fig. 1). It was drained several times a week but production of cyst fluid continued. The cyst fluid was rich in proteins, lipids, and cells. The cells were mostly granulocytes, but there were also many phagocytes. Some atypical cells were also found but their origin could not be established and the analysis did not result in a specific diagnosis.

During this time many cycles of compression and decompression were monitored by tests of visual acuity and of the visual field. The pattern of visual loss upon increasing interference with optic nerve conduction was remarkably similar during all episodes (Fig. 2). The first sign of expansion of the cyst was slight reduction in visual acuity. A measurable change in the visual field was not seen until acuity had dropped from 1.0 to approximately 0.6 . The field change consisted of an increased depression of the previously damaged lower nasal quadrant, in the form of a nasally expanding sector scotoma pointing towards the fixation point. With increasing filling of the cyst, the scotoma expanded towards the lower: vertical meridian and the intermediary isopter was split into two parts, delineating a paracentral and a temporal area respectively. At the same time a concentric contraction of the peripheral field developed. Peripheral depression might have been detected earlier had we used a smaller target rather than the $V / 48$ object of the Goldmann perimeter. Progressive de pression occurred until only small nasal and teme poral islands of vision remained. Acuity at this stage? was reduced to counting fingers. If cyst filling progressed, the right eye went completely blind and a small upper temporal depression appeared in the contralateral visual field.

The rate of cyst filling increased until puncture had to be made almost daily. It was then decided to try local irradiation. ${ }^{90} \mathrm{Y}$ was instilled into the cyst via the catheter, to give a calculated dose of approximately $30,000 r$. This resulted in accelerated production of fluid and, in spite of several drains daily, vision could not be restored to the pre-injection level. This was thought to depend on oedema of the cyst wall. An attempt to control this situation was made by injecting $1 \mathrm{ml}$ Zenker's solution into the cyst. Thirty seconds later the cyst was rinsed repeatedly with saline. The right eye then went blind and the left eye presented an absolute temporal hemianopia. Acuity was not affected on the left. Surgical exploration two days later showed scar tissue covering the chiasm and optic nerves, but no discolouration was seen. The tumour cyst could not be identified.

There were no signs whatsoever of recurrence during the following four years. 

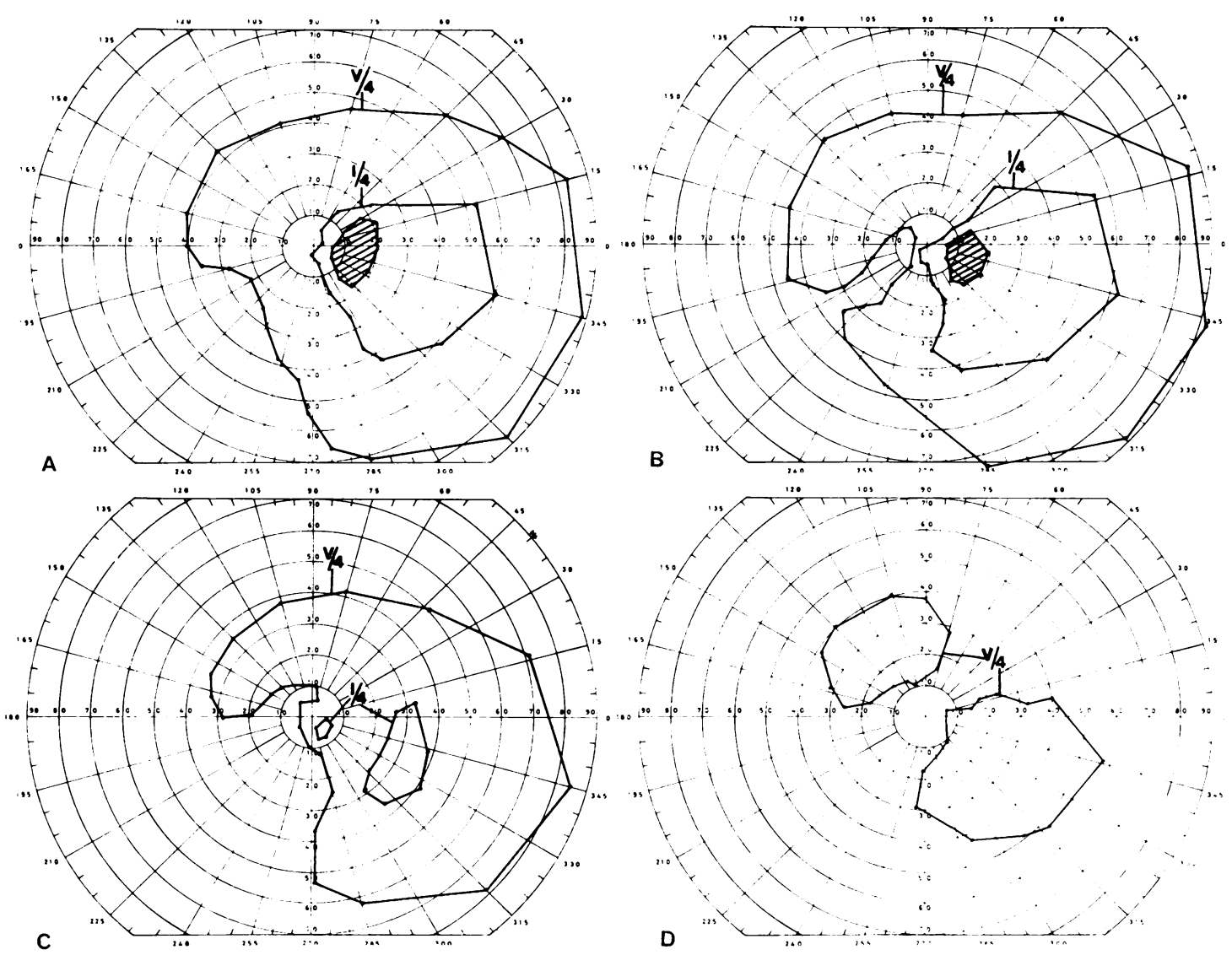

FIG. 2 Cycle of field changes caused by expansion of suboptic craniopharyngioma cyst on the right. A: partial optic atrophy with nasal field depression and contraction reflects pre-existing, irreversible damage. Visual acuity 1.0. B: start of sector scotoma within previously defective visual field area. Acuity 0.6. C: increasingly defective nasal visual field, with signs of early depression also in temporal area. Acuity 0.5. D: nasal and temporal remnants. Acuity: counting fingers. Upon drainage of reservoir, vision was immediately restored to the stage depicted in A.

\section{DISCUSSION}

It appears reasonable to summarise the sequence of field change as an increasingly severe uniform depression and to attribute the changes localised in the lower nasal quadrant to pre-existing damage to optic nerve axons serving this area. Such damage is evidenced by persistent lower nasal contraction and nasal foreshortening of the intermediary isopter after cyst drainage. The slight pallor of the optic disc, which was noted before the very first operation, also indicates a loss of nerve fibres.

Uniform depression of the visual field presumably reflects a uniform distribution across the optic nerve of conduction failure. In this situation it is worthy of note that, although we had access to reliable base-line field data, we were unable to detect a change in the visual field before acuity had dropped to approximately 0.6. Similar observations were made by Knight et al. (1972) in a series of cases with early compression of one optic nerve.

The fact that acuity drops before there is a measurable change in the visual field is often attributed to a greater vulnerability of macular fibres. Resolution and light sensitivity differ too much as functions to be directly compared, however. If we had had access to comparable methods in testing the macular and the peripheral function it might have been possible to detect disturbances of the two at the same time.

Knight et al. (1972) stressed deficient colour vision as an early sign of conduction disturbance. Because 
of pre-existing defects in colour vision, we were unable to explore this particular function in our patient.

Symptoms and signs recurred at an ever increasing rate, from three times a year to twice a day. The time course and duration of impairment did not influence the pattern of functional loss or the rate of recovery upon cyst drainage. Recovery was too fast to allow identification of the specific nature of recovery. The patient was also unable to analyse accurately different phases during recovery of conduction: subjectively, vision returned to normal instantaneously. This rapid recovery was observed more than 50 times. Earlier reports on the rate of recovery upon relief of compression of the anterior visual pathways do not emphasise such a dramatic normalisation of function. Although it is a commonplace clinical observation that patients may recover vision within perhaps a day after surgery (Gregorius et al., 1975; Kayan and Earl, 1975) the circumstances surrounding surgery rarely if ever allow formal testing of a fully alert patient at the precise moment when compression is relieved. Studies of the visual evoked response during surgery for suprasellar meningioma attest to the possibilities of an extremely rapid recovery of conduction (Feinsod and Auerbach, 1971; Feinsod et al., 1976). Our case proves that functional recovery may be very much faster than previously thought possible.

Our patient's remarkable tolerance to repeated and protracted optic nerve compression, and her uniquely rapid recovery upon release of cyst distension, may help to illuminate the roles of various pathophysiological mechanisms involved in the production of visual failure with so-called compressive lesions of the optic nerve.

Conduction block may obviously occur repeatedly without inflicting irreversible damage to the optic nerve fibres. What functional and structural alterations cause a reversible conduction block in the anterior visual pathway are poorly understood. Ischaemia, compression, and mechanical deformation are factors traditionally thought to be involved. O'Connell (1973) recently has emphasised tension as an important causative factor in bitemporal hemianopia due to a suprasellar mass. A more or less severe disturbance of intraneural microcirculation is presumably unavoidable in mechanical deformation and compression. The latter factors may also be capable of disturbing the continuous transport of macromolecules and organelles that occurs in the optic nerve (Obstbaum and Podos, 1974; Wirtschafter et al., 1975). It is known that axonal flow within the intraocular part of the optic nerve fibres is partially or totally blocked when the intraocular pressure is raised artificially (Anderson and Hendrickson, 1974; Levy, 1974). Axonal transport may recover upon normalisation of the intraocular pres- sure (Levy, 1974). Optic nerve fibres may well survive several weeks of chemical block (colchicine, vinblastine) of axonal flow (Karlsson et al., 1971; Bunt, 1973). There is little, if any, change in optic nerve conduction in this situation, although synaptic transmission may fail a few days after chemical block (Peresić and Cuénod, 1972). The findings from these experimental studies suggest that a block of axonal transport is not a major factor in the production of rapidly reversible conduction block.

Compression and mechanical deformation may also induce segmental demyelination. Repair processes may explain the protracted functional recovery (within weeks) characteristic of moderate compression blocks in peripheral nerves (Agayo et al., 1971; Ochoa et al., 1972). A similarly protracted recovery of the anterior visual pathway is not unusual after surgical decompression of the optic chiasm (Nover, 1962). The time course of improvement in our patient speaks strongly against such factors in her recovery.

Recent experimental studies on peripheral nerves have shown that a local supply of energy to a nerve ise needed to support both nerve conduction and axonalo transport and that anoxia rapidly blocks both func:tions (Ochs, 1974). Conduction and flow resume equally rapidly if energy supply is restored within 1.5 hours. With more prolonged anoxia only conduction resumes rapidly (Ochs, 1974). The instantaneous recovery of vision in our patient upon release of cyst fluid favours the interpretation that the conductiono block was due mainly to an ischaemic interruption of local energy supply, presumably induced by meche anical compression and deformation.

Dr H. Okamoto was helpful in translating the paper by Isayama (1970).

\section{REFERENCES}

Agayo, A., Nair, C. P. V., and Midgley, R. (1971). Experimental progressive compression neuropathy in the rabbit. Archives of Neurology (Chic.), 24, 358-364.

Anderson, D. R., and Hendrickson, A. (1974). Effect of intraocular pressure on rapid axoplasmic transport in monkey optic nerve. Investigative Ophthalmology, 13, 771-783.

Bunt, A. H. (1973). Effects of vinblastine on microtubule structure and axonal transport in ganglion cells of the rabbit retina. Investigative Ophthalmology, 12, 579-590.

Feinsod, M., and Auerbach, E. (1971). The electroretinogram and the visual evoked potential in two patients with tuberculum sellae meningioma before and after decompression of the optic nerve. Ophthalmologica, 163, 360-368.

Feinsod, M., Selhorst, J. B., Hoyt, W. F., and Wilson, 
C. B. (1976). Monitoring optic nerve function during craniotomy. Journal of Neurosurgery, 44, 29-31.

Gregorius, F. K., Hepler, R. S., and Stern, W. E. (1975). Loss and recovery of vision with suprasellar meningiomas. Journal of Neurosurgery, 42, 69-75.

Isayama, Y. (1970). Changes in visual field associated with changes of tension in large cyst, which is craniopharyngioma, extending to the middle cranial fossa. Acta Societas Ophthalmologica Japonica, 74, 596-604. (In Japanese.)

Karlsson, J-O., Hansson, H-A., and Sjöstrand, J. (1971). Effect of colchicine on axonal transport and morphology of retinal ganglion cells. Zeitschrift für Zellforschung und Mikroskopische Anatomie, 115, 265-283.

Kayan, A., and Earl, C. J. (1975). Compressive lesions of the optic nerve and chiasm. Pattern of recovery of vision following surgical treatment. Brain, 98, 13-28.

Knight, C. L., Hoyt, W. F., and Wilson, C. B. (1972). Syndrome of incipient prechiasmal optic nerve compression. Archives of Ophthalmology, 87, 1-11.

Levy, N. S. (1974). The effects of elevated intraocular pressure on slow axonal protein flow. Investigative Ophthalmology, 13, 691-695.
Nover, A. (1962). Über das Verhalten des Opticus nach längerdauernder Kompression. Fortschritte der Neurologie, Psychiatrie und ihren Grenzgebiete, 30, 228-233.

Obstbaum, S. A., and Podos, S. M. (1974). Axoplasmic transport. Investigative Ophthalmology, 13, 81-84.

Ochoa, J., Fowler, T. J., and Gilliatt, R. W. (1972). Anatomical changes in peripheral nerves compressed by a pneumatic tourniquet. Journal of Anatomy, 113, $433-455$.

Ochs, S. (1974). Energy metabolism and supply of $\sim P$ to the fast axoplasmic transport mechanisms in nerve. Federation Proceedings, 33, 1049-1058.

O'Connell, J. E. A. (1973). The anatomy of the optic chiasma and heteronymous hemianopia. Journal of Neurology, Neurosurgery, and Psychiatry, 136, 710-723.

Pereśić, M., and Cuénod, M. (1972). Synaptic transmission depressed by colchicine blockade of axoplasmic flow. Science, 175, 1140-1142.

Wirtschafter, J. D., Rizzo, F. J., and Smiley, B. C. (1975). Optic nerve axoplasm and papilledema. Survey of Ophthalmology, 20, 157-189. 